\title{
Plastrum Testudinis Extracts Promote NSC Differentiation into Dopaminergic Neuron by Regulating the Interaction of TET1 and FoxA2
}

\author{
Jun Zhong $\mathbb{D}^{1,2}$ Sen Ye, ${ }^{1,2}$ Xiaoli Zhou, ${ }^{3}$ Jiapei Huang, ${ }^{1,2}$ Xican Li, ${ }^{4}$ Saixia Zhang, \\ Jianhong Zhou, ${ }^{5}$ Dongfeng Chen, ${ }^{1}$ and Caixia $\mathrm{Li} \mathbb{C}^{1,2}$ \\ ${ }^{1}$ School of Basic Medical Sciences, Guangzhou University of Chinese Medicine, Guangzhou, Guangdong Province, China \\ ${ }^{2}$ Research Center of Integrative Medicine, School of Basic Medical Sciences, Guangzhou University of Chinese Medicine, \\ Guangzhou, Guangdong Province, China \\ ${ }^{3}$ NangFang College of Sun Yat-Sen University, Guangzhou, Guangdong Province, China \\ ${ }^{4}$ School of Pharmaceutical Sciences, Guangzhou University of Chinese Medicine, Guangzhou, Guangdong Province, China \\ ${ }^{5}$ The Center of Laboratory Animal, Guangzhou University of Chinese Medicine, Guangzhou, Guangdong Province, China \\ Correspondence should be addressed to Caixia Li; 020691@gzucm.edu.cn
}

Received 30 November 2019; Revised 24 February 2020; Accepted 6 March 2020; Published 20 April 2020

Guest Editor: Rômulo Dias Novaes

Copyright (c) 2020 Jun Zhong et al. This is an open access article distributed under the Creative Commons Attribution License, which permits unrestricted use, distribution, and reproduction in any medium, provided the original work is properly cited.

\begin{abstract}
In recent years, stem cells have gained much attention for the treatment of neurodegenerative diseases. However, inducing neural stem cell directionally differentiation is a difficult problem in the treatment of Parkinson's disease (PD) by stem cell therapy. Plastrum Testudinis (PT) can enhance the number of TH-positive neurons in the PD rat brain substantia nigra, but the underlying mechanism has not been clarified. Here, we aimed at further investigating the mechanism by which PT can promote NSC differentiation into dopaminergic neurons. A rat model of PD was used for detecting the effect of PT on the rat brain substantia nigra in vivo. The results showed the expressions of tyrosine hydroxylase (TH) and TET1 enzyme were increased after treatment with PT. Consequently, Plastrum Testudinis extracts (PTEs) were used for inducing NSC differentiation into dopaminergic neurons ex vivo. During differentiation of NSCs induced by PTE, TH expression was increased, with a concomitant increase in both TET1 and FoxA2. Next, we performed coimmunoprecipitation analysis to examine the interaction between TET1 protein and FoxA2 protein. Our results show that PTE can increase the binding rate of TET1 and FoxA2. Thus, our findings show that PTE can increase the efficiency of NSCs to directionally differentiate into dopaminergic neurons and provide experimental evidence for $\mathrm{PT}$ in the treatment of Parkinson's disease.
\end{abstract}

\section{Introduction}

Parkinson's disease (PD) is a common progressive neurodegenerative disease that occurs in middle-aged and elderly people. Its main pathological change is degeneration and deletion of dopaminergic neurons in the substantia nigra, which leads to a series of clinical symptoms such as resting tremor, bradykinesia, muscle rigidity, and autonomic dysfunction. Because of the aging population, medical expenditure for PD (which is one of the diseases with the highest incidence of nervous system diseases) can be a considerable problem for the socioeconomic burden in the future [1]. Unfortunately, the pathogenesis of PD is still not clear. Until recently, levodopa was the main drug used for the treatment of PD. However, long-term use of levodopa is associated with the development of levodopa-induced motor complications [2]. Accordingly, some studies have suggested using cell replacement therapy as an alternative direction for the treatment of PD [3, 4].

Neural stem cells (NSCs) are the cell population of the nervous system with the potential for self-renewal and multidirectional differentiation, which could differentiate 
into neurons, astrocytes, and oligodendrocytes [5]. Researchers have shown that NSCs have good application prospects for the treatment of age-related neurodegenerative diseases [6]. Moreover, these studies have demonstrated that NSCs can differentiate into dopaminergic neurons and may reverse the degeneration process of PD by replacing the loss of dopaminergic neurons. Consequently, inducing directed differentiation of NSCs into dopaminergic neurons has become a key issue.

Studies have shown that DNA demethylation is indispensable for the differentiation of NSCs $[7,8]$. Teneleven translocation 1 (TET1) is a member of the TET family that is highly expressed in embryonic stem cells and the nervous system and is an important enzyme in DNA demethylation [9]. When DNA demethylation occurs, TET1 converts 5-methylcytosine $(5 \mathrm{mC})$ to 5-hydroxymethylcytosine $(5 \mathrm{hmC})$ under the combined action of iron(II) and $\alpha$-ketoglutarate acid-dependent dioxygenases [10]. Interestingly, $5 \mathrm{hmC}$ is a biomarker of DNA demethylation that is highly enriched in the brain and crucial during neural development [11]. Nevertheless, the mechanism by which DNA demethylation regulates NSC differentiation has not been clarified, yet many studies had revealed a relationship [12, 13]. Hahn et al. demonstrated that $5 \mathrm{hmC}$ levels were increased during neuronal differentiation in mouse brain and were associated with activation of genes important for related neuronal functions. Additionally, TET1 plays a key role in the transformation of human fibroblasts into induced dopaminergic neurons [14]. Recently, a study reported that liver progenitor cell differentiation requires TETs and forkhead box A2 (FoxA2), providing specific direction for our research [15]. The FoxA2 protein is a member of the forkhead family, with a wide range of expression in the midbrain. Further, FoxA2 is an essential nuclear transcription factor involved in the differentiation of NSCs into dopaminergic neurons [16]. One study demonstrated that deletion of FoxA2 causes a reduction in the number of TH-positive midbrain dopaminergic (mDA) neurons [17]. TH catalyzes the formation of L-dopa by tyrosine and is the rate-limiting enzyme of catecholamine synthesis. Further, TH has been used as a biomarker of dopaminergic neurons [18]. For all of the above reasons, we are interested in increasing the efficiency of the directional differentiation of NSCs into dopaminergic neurons.

Traditional Chinese medicine (TCM) can regulate the differentiation of NSCs by changing the microenvironment. Many studies have suggested that single TCM, extracts of TCM, and some TCM monomers can regulate differentiation of NSCs [19-21]. Plastrum Testudinis (PT; Carapax Testudinis, Guijia) is a TCM that has been shown to have potential in promoting osteogenic differentiation of bone marrow stem cells [22]. However, the mechanism by which PT induces NSC differentiation into dopaminergic neurons is not known precisely. Previous research from our group identified the active component of PT as ethyl acetate extracts from PT (i.e., PTE). In this study, we further investigated the mechanism by which PTE promotes NSC differentiation into dopaminergic neurons.

\section{Experimental Procedure}

2.1. Rat Model of Parkinson's Disease. This study was carried out in accordance with national animal experiment protocols and approved by the Experimental Animal Ethics Committee of Guangzhou University of Traditional Chinese Medicine (Protocol no. 20130035). All surgeries were performed under sodium pentobarbital anesthesia, and all efforts were made to minimize suffering.

The rat model was established by injecting 6-hydroxydopamine (6-OHDA) into the midbrain, according to the method used in a study [23]. Sprague Dawley (SD) male rats weighing 90-100 g were selected, anesthetized with $1 \%$ pentobarbital sodium, and fixed with a stereotaxic apparatus. The $6-\mathrm{OHDA}$ was diluted in $0.9 \% \mathrm{NaCl}$ solution. Taking the midpoint of the coronal suture as the origin, the height $+7.8 \mathrm{~mm}$, the ordinate $-5.2 \mathrm{~mm}$, and the abscissa $+1.8 \mathrm{~mm}$ is the substantia nigra $(\mathrm{SN})$; the height $+7.8 \mathrm{~mm}$, the ordinate $-4.6 \mathrm{~mm}$, and the abscissa $+0.9 \mathrm{~mm}$ is the substantia nigra pars compacta $(\mathrm{SNpc})$. The left $\mathrm{SN}$ and $\mathrm{SNpc}$ of rats were injected with $8 \mu \mathrm{l} 6$-OHDA $(4 \mu \mathrm{g} / \mu \mathrm{l})$, respectively. And, at the two injection points in the sham operation group, the same volume of $0.9 \% \mathrm{NaCl}$ solution was injected. One week after operation, rats were tested with apomorphine $(0.5 \mathrm{mg} /$ $\mathrm{kg}$ ) by intraperitoneal injection once a week, and the number of rotations of the rats within $30 \mathrm{~min}$ was observed and recorded. The average rotational speed of more than $7 \mathrm{r} / \mathrm{min}$ was regarded as a successful PD rat model. The successful rat models were randomly divided into four groups: PD model group, positive drug group, low-dose drug group, and highdose drug group, while the wild-type rats were randomly divided into the blank control group and the sham operation group. Each drug group was treated continuously for 45 days. The complete animal groupings and drug treatment measures are as follows: blank control group: normal SD rats without surgery, $1 \mathrm{ml} / \mathrm{kg} 0.9 \% \mathrm{NaCl}$ solution per day by intragastric administration for each rat; sham operation group: the same volume of $0.9 \% \mathrm{NaCl}$ solution was used instead of 6-OHDA during operation, and $1 \mathrm{ml} / \mathrm{kg} 0.9 \%$ $\mathrm{NaCl}$ solution per day by intragastric administration for each rat; PD model group: $1 \mathrm{ml} / \mathrm{kg} 0.9 \% \mathrm{NaCl}$ solution per day by intragastric administration for each rat; positive drug group: $1 \mathrm{ml} / \mathrm{kg}$ positive drug solution per day by intragastric administration for each rat $(10 \mathrm{mg} / \mathrm{kg}$ levodopa $+2.5 \mathrm{mg} / \mathrm{kg}$ benserazide); low-dose drug group: $0.4 \mathrm{ml}$ PT $(1.1 \mathrm{~g} / \mathrm{d})$ per day by intragastric administration for each rat; and highdose drug group: $3.4 \mathrm{ml}$ PT $(9.9 \mathrm{~g} / \mathrm{d})$ per day by intragastric administration for each rat.

\subsection{Immunohistochemical Staining Assay. Rats were sacri-} ficed by overinjecting $1 \%$ pentobarbital sodium (at least $>150 \mathrm{mg} / \mathrm{kg}$ ), and the brain tissues were fixed in $4 \%$ paraformaldehyde. Brain tissues were embedded in paraffin, and the tissues were sectioned to a thickness of $4 \mu \mathrm{m}$. Staining of rat brain (substantia nigra) tissue sections was performed. The sections were deparaffinized by xylene and rehydrated by gradient ethanol solution (from $100 \%$ to $75 \%$ ). Sections were inhibited for endogenous peroxidase activity using $3 \%$ 
$\mathrm{H}_{2} \mathrm{O}_{2}$ buffer at $37^{\circ} \mathrm{C}$ for $15 \mathrm{~min}$. The antigen retrieval was done by using a microwave oven with citric acid solution (pH 6.0) at $100^{\circ} \mathrm{C}$ for $10 \mathrm{~min}$, and then, the slices were allowed to cool sufficiently and equilibrated to room temperature. Sections were permeabilized by $0.5 \%$ Triton X-100 (diluted in PBS pH 7.4, PBS was purchased from Gibco, \#C10010500BT) for $15 \mathrm{~min}$ at $37^{\circ} \mathrm{C}$ and blocked in $10 \%$ goat serum for $30 \mathrm{~min}$ at $37^{\circ} \mathrm{C}$. Incubation with primary antibodies (polyclonal rabbit anti-tyrosine hydroxylase, 1:500, Abcam, \# ab112; polyclonal rabbit anti-TET1, 1:100, Abcam, \# ab191698; monoclonal rabbit anti-FoxA2, $1: 200$, Abcam, \#ab108422; polyclonal rabbit anti-5-hydroxymethylcytosine, 1:200, Active Motif, \# 39769) was performed overnight at $4^{\circ} \mathrm{C}$ and secondary antibody (biotinylated goat anti-mouse/rabbit IgG, Boster, \#SA1020) was incubated at room temperature for 1 hour. Subsequently, streptavidin-biotin complex (SABC kit, Boster, \#SA1020) was incubated at room temperature for $30 \mathrm{~min}$, color development was observed by adding diaminobenzidine (DAB color development kit, Boster, \#AR1022) for $10 \mathrm{~min}$, and hematoxylin is used for counterstaining. The sections were imaged using Olympus image analysis system and analyzed using ImageJ software.

\subsection{Preparation of Extracts from Plastrum Testudinis. PT and} PTE were provided by Professor Xican Li, from the School of Pharmaceutical Sciences at Guangzhou University of Chinese Medicine. PTE was obtained based on the previously established methods [24, 25]. The part of ethyl acetate solvents was recovered and was dissolved in dimethyl sulphoxide $(30 \mathrm{mg} / \mathrm{mL})$.

2.4. Neural Stem Cells Isolated and Cultured. SD rats of E1417 were provided from the Animal Center of Guangzhou University of Traditional Chinese Medicine. Neural stem cells were isolated from the embryonic brain of E14-17 SD rat according to a report [26]. Embryonic brain tissues were mechanically dissociated and digested by $0.25 \%$ trypticEDTA (Gibco). After digestion was completed, cells were cultured with serum-containing medium (DMEM/F12 1:1) supplemented with $10 \%$ fetal bovine serum to terminate the digestion and washed twice with phosphate-buffered saline (PBS). The single-cell suspension was prepared to use the blow of Pasteur pipette with based growth medium, which is a serum-free medium (DMEM/F12 1:1) supplemented with the growth factor Fibroblast Growth Factor-basic and Epidermal Growth Factor at $20 \mathrm{ng} / \mathrm{ml}$ (all from GenScript) as well as the $2 \%(\mathrm{v} / \mathrm{v}) \mathrm{B} 27,100$ units/ml penicillin, and $100 \mu \mathrm{g} / \mathrm{ml}$ streptomycin (all from Gibco). The cells were seeded into $75 \mathrm{ml}$ culture flasks and cultured at $37^{\circ} \mathrm{C}$ in an environment of $5 \% \mathrm{CO}_{2}$. The culture medium was changed every 3 days.

2.5. Induction and Differentiation of NSCs. When NSCs were at passage 2 , single cell suspension was prepared by mechanical dissociation. Cells were plated into 6-well culture plates and cultured with serum-containing medium $(10 \%$
FBS in DMEM/F12) supplemented with 100 units/ml penicillin and $100 \mu \mathrm{g} / \mathrm{ml}$ streptomycin, and the PTE $(3 \mu \mathrm{g} /$ $\mathrm{ml}, 30 \mu \mathrm{g} / \mathrm{ml}$ ) was immediately added to induce NSC differentiation. The culture medium was changed every 2 days. At 5 days of differentiation, TET1, FoxA2, global 5hmC levels, and $\mathrm{TH}$ expression were assessed.

2.6. Western Blot Analysis. Total proteins were lysed in RIPA lysis and extraction buffer (Thermo) with Protease Inhibitor Cocktail (EDTA-Free, 100X in DMSO (Bimake)) for $15 \mathrm{~min}$ on ice. Nuclear proteins were extracted by using Nuclear and Cytoplasmic Protein Extraction Kit (KeyGEN) according to the manufacturer's instructions. Protein concentration was quantified by BCA protein assay kit (FD) according to the manufacturer's instructions. The proteins were used for western blotting, separated by $10 \%$ SDS-PAGE (Beyotime), and then transferred to $0.45 \mu \mathrm{m}$ polyvinylidene fluoride (PVDF) membrane (Millipore). The membranes were blocked by $5 \%$ nonfat dry milk (Cell Signaling Technology) for 2 hours at room temperature. Primary antibodies were incubated overnight at $4^{\circ} \mathrm{C}$, and secondary antibodies were incubated for 1 hour at room temperature, and the signals were detected by Immobilon Western Chemiluminescent HRP Substrate (Millipore). The following antibodies were used: rabbit anti-TET1 antibody (1: 1000, polyclonal, Abcam, Cambridge, UK, no. ab191698), mouse anti-FoxA2 antibody (1:500, monoclonal, Abcam, Cambridge, UK, no. ab60721), rabbit anti-FoxA2 antibody (1: 1000, monoclonal, Abcam, Cambridge, UK, no. ab108422), rabbit anti-tyrosine hydroxylase antibody $(1: 500$, polyclonal, Abcam, Cambridge, UK, no. ab112), mouse anti- $\beta$-actin $(1: 500$, monoclonal, Boster Biological Technology, Wuhan, CN, no. BM0627), rabbit anti-Lamin B1 (1:100, polyclonal, Boster Biological Technology, Wuhan, CN, no. PB0640), goat anti-rabbit IgG H\&L (HRP) $(1: 10000$, polyclonal, Abcam, Cambridge, UK, no. ab6721), and rabbit anti-mouse IgG H\&L (HRP) (1:10000, polyclonal, Abcam, Cambridge, UK, no. ab6728).

2.7. Dot Blot Analysis. The genomic DNA samples were extracted by Takara MiniBEST Universal Genomic DNA Extraction Kit Ver.5.0 (Takara, Shiga, JP, no. 9765). The PVDF membrane was activated with methanol for $5 \mathrm{~min}$. After the membrane dried, DNA samples were spotted onto the PVDF membrane, and then the membrane was left to dry at $60^{\circ} \mathrm{C}$ for 2 hours. The membranes were blocked by $5 \%$ bovine serum albumin for 2 hours at room temperature. The primary antibodies were incubated overnight at $4^{\circ} \mathrm{C}$, and the secondary antibody was incubated for 2 hours at room temperature, and the signals were detected by Immobilon Western Chemiluminescent HRP Substrate (Millipore). The following antibodies were used: rabbit anti-5-hydroxymethylcytosine $(1: 10000$, polyclonal, Active Motif, Carlsbad, USA, no. 39769) and goat anti-rabbit IgG H\&L (HRP) (1 : 10000, polyclonal, Abcam, Cambridge, UK, no. ab6721).

2.8. Real-Time Quantitative PCR ( $q R T-P C R$ ) Analysis. NSCs were plated into 6-well culture plates and cultured with the PTE $(30 \mu \mathrm{g} / \mathrm{ml})$ for 5 days. Total RNA was extracted 
from different groups of cells by TRIzol reagent (Invitrogen) and Direct-zol ${ }^{\text {TM }}$ RNA MiniPrep Kit (ZYMO) according to the manufacturer's instructions. About $2 \mu \mathrm{g}$ total RNA was reverse-transcribed into $\mathrm{cDNA}$ by PrimeScript ${ }^{\mathrm{TM}} \mathrm{RT}$ reagent Kit (Takara, Shiga, JP, no. RR037A) according to the manufacturer's instructions, which is used for qRT-PCR. qRT-PCR was performed by using TB Green ${ }^{\mathrm{TM}}$ Premix Dimer Eraser $^{\mathrm{TM}}$ kit (Takara, Shiga, JP, no. RR820A) and following the protocol: initial denaturation at $95^{\circ} \mathrm{C}$ for $30 \mathrm{sec}$, 40 cycles of PCR followed by $95^{\circ} \mathrm{C}$ for $5 \mathrm{sec}, 58^{\circ} \mathrm{C}$ for $30 \mathrm{sec}$, and $72^{\circ} \mathrm{C}$ for $1 \mathrm{~min}$, and the relative expressions of different genes were quantitatively analyzed by the $2^{-\Delta \Delta \mathrm{Cq}}$ method. Gapdh gene was used as a housekeeping gene for mRNA expression. The primers are shown in Table 1.

2.9. Immunofluorescence Staining Assay. NSCs were plated into 24-well culture plates with cell climbing slices, and cells were cultured with PTE $(30 \mu \mathrm{g} / \mathrm{ml})$ for 5 days. Cells were washed twice with PBS, fixed in $4 \%$ paraformaldehyde for $20 \mathrm{~min}$ at room temperature, permeabilized by $0.5 \%$ Triton $\mathrm{X}-100$ for $15 \mathrm{~min}$ at $37^{\circ} \mathrm{C}$, and blocked in $10 \%$ goat serum for $30 \mathrm{~min}$ at $37^{\circ} \mathrm{C}$. The primary antibodies were incubated overnight at $4^{\circ} \mathrm{C}$ and the secondary antibody was incubated at room temperature for 1 hour. Cells were incubated with $4^{\prime}$,6-diamidino-2-phenylindole (DAPI) for $5 \mathrm{~min}$ and mounted by antifade mounting medium. The positive expression of cells was observed by using laser scanning confocal microscopy and the image was taken by ZEN 2 software. The following antibodies were used: rabbit antiTET1 antibody $(1: 1000$, polyclonal, Abcam, Cambridge, UK, no. ab191698), mouse anti-FoxA2 antibody (1:500, monoclonal, Abcam, Cambridge, UK, no. ab60721), rabbit anti-tyrosine hydroxylase antibody (1:500, polyclonal, Abcam, Cambridge, UK, no. ab112), mouse anti-5hydroxymethylcytosine $(1: 10000$, monoclonal, Active Motif, Carlsbad, USA, no. 39999), DAPI (1:1000, Beyotime, Shanghai, CN, no. C1002), goat anti-rabbit IgG H\&L (Alexa Fluor 488) (1:1000, polyclonal, Abcam, Cambridge, UK, no. ab150077), and goat anti-mouse IgG H\&L (Alexa Fluor 647) ( 1 : 1000, polyclonal, Abcam, Cambridge, UK, no. ab150119).

2.10. Coimmunoprecipitation (Co-IP) Analysis. NSCs were plated into $100 \times 100 \mathrm{~mm}$ cell culture dish and differentiation was induced by PTE $(30 \mu \mathrm{g} / \mathrm{ml})$ for 5 days. Coimmunoprecipitation analysis was performed by using Pierce $^{\mathrm{TM}}$ Crosslink Magnetic IP/Co-IP Kit (Thermo) according to the manufacturer's instructions. Briefly, the first is binding of antibody to protein $A / G$ magnetic beads and the final concentration of antibody (mouse anti-FoxA2 antibody, monoclonal, Abcam, Cambridge, UK, no. ab60721) was $5 \mu \mathrm{g}$ per sample. Then, the bound antibody was crosslinked, and the antibody-crosslinked beads were stored at $4^{\circ} \mathrm{C}$. Cell protein was extracted, and the protein concentration was determined. Finally, the protein was added to the antibodycrosslinked beads for immunoprecipitation and the beads and proteins were magnetically separated, and the proteins were detected by western blot analysis.
2.11. siRNA Transfection. siRNA silencing of Tet1 or Foxa2 was performed. The silencing fragments of Tet1 and Foxa2 were designed and synthesized by RIBOBIO Company (Guangzhou, CN.). Single cell suspension was prepared by mechanical dissociation. Cells were plated into 6-well culture plates and cultured with serum-containing medium (10\% FBS in DMEM/F12). When the cells were grown to $70 \%-90 \%$, siRNA was transfected into cells by Lipofectamine ${ }^{\circledR} 2000$ reagent (Invitrogen) according to the manufacturer's instructions, and the final concentration of transfected siRNA was $50 \mathrm{nM}$. After siRNA was transfected for $24 \mathrm{~h}$, the expression of Tet 1 and Foxa 2 was assessed by qRT-PCR, and the efficiency of silencing was evaluated; the siRNA fragment with the best silencing efficiency was selected for subsequent experiments.

siRNA was transfected for $24 \mathrm{~h}$, and cells were cultured with PTE $(30 \mu \mathrm{g} / \mathrm{ml})$ for $24 \mathrm{~h}$. The expression of Th gene mRNA level was analyzed by using qRT-PCR.

2.12. Statistical Analyses. Data in this study were expressed as mean \pm standard deviation. Data analysis was done by using GraphPad Prism7 software, at least three independent experiments in each group. The data of in vivo experiment were analyzed using two-way ANOVA and Tukey's test for multiple comparisons; $t$-test (two-tailed) was used for the comparison between two sample groups. The $P$ value $<0.05$ was considered as a significant difference.

\section{Results}

3.1. Effect of PT on a Rat Model of PD. We established a rat model of PD to determine if PT can induce differentiation of NSCs into dopaminergic neurons. We detected TH expression in brain tissue sections and observed $\mathrm{TH}$-positive cells in the model group. The results showed a significant reduction compared with the control group (Figure 1(a)). Simultaneously, we observed a significantly higher number of positive cells in the drug groups compared with the PD model group (Figures $1(\mathrm{a})$ and $1(\mathrm{~b})$ ). To examine the mechanisms of DNA demethylation in the rat PD model, we detected the expression of $5 \mathrm{hmC}$ in brain tissue sections. The results showed significant downregulation in the PD model group compared with the control group. Further, both the high-dose drug and low-dose drug groups showed significant upregulation compared with the PD model group (Figures 1(c) and 1(d)). Additionally, we detected expression of TET1, as the rate-limiting enzyme of $5 \mathrm{hmC}$ production. We observed that the variable trend of TET1 expression was consistent with $5 \mathrm{hmC}$ production. Compared with the control group, TET1 expression was decreased in the PD model group, while the high-dose drug and low-dose drug groups showed a significant increase compared with the PD model group (Figures 1(e) and 1(f)).

3.2. Effect of PTE on Differentiation of NSCs. To investigate the mechanism by which PT induces differentiation of NSCs into dopaminergic neurons, NSCs were differentiated ex vivo using PTE. 
TAble 1: Primers for qRT-PCR.

\begin{tabular}{|c|c|c|}
\hline Gene & Sequence & Supplier \\
\hline Gapdh & $\begin{array}{l}\text { Forward: 5'-GTTCAACGGCACAGTCAAGG-3' } \\
\text { Reverse: } 5^{\prime} \text {-GACGCCAGTAGACTCCACGAC-3' }\end{array}$ & \multirow{5}{*}{ Sangon Biotech, Shanghai, CN } \\
\hline Th & Forward: 5'-ACTGTGGCTACCGAGAGGAC-3' & \\
\hline in & $\begin{array}{c}\text { Reverse: } 5^{\prime} \text {-AATCACGGGCGGACAGTAGA-3' } \\
\text { Forward: } 5^{\prime} \text {-CAGGAGAAACGCATGGTACAACAGA-3' }\end{array}$ & \\
\hline Tet 1 & Reverse: $5^{\prime}$-CGCTTGCTTGTGTATGGAGTTGG-3' & \\
\hline Foxa2 & $\begin{array}{l}\text { Forward: 5'-ACGAACTGGCGTTGAAGGAAG-3' } \\
\text { Reverse: } 5^{\prime} \text {-CTGAACCTGAGAAGCCTGTGTC-3' }\end{array}$ & \\
\hline
\end{tabular}

NSCs were cultured with serum-containing medium and treated with PTE $(0 \mu \mathrm{g} / \mathrm{mL}, 3 \mu \mathrm{g} / \mathrm{mL}$, and $30 \mu \mathrm{g} / \mathrm{mL})$ for 5 days, and then cell proteins were extracted for western blotting analysis. We detected the expression of TH, which is a dopaminergic biomarker. Our results showed that $\mathrm{TH}$ expression was not changed in the $3 \mu \mathrm{g} / \mathrm{mL}$ PTE group compared with the control group $(0 \mu \mathrm{g} / \mathrm{mL})$. Further, TH expression was significantly increased in the $30 \mu \mathrm{g} / \mathrm{mL}$ PTE group (Figure 2(a)). Consequently, we selected a dose of $30 \mu \mathrm{g} / \mathrm{mL}$ for further experiments. Corroboratively, mRNA levels of the Th gene were consistent with the trend observed with TH protein levels, with significantly higher Th mRNA levels in the PTE group compared with the control group (Figure 2(b)). Immunofluorescence showed that PTE increased the number of $\mathrm{TH}$-positive neurons in differentiated NSCs. In NSC culture not containing PTE, only a few cells were spontaneously differentiated into $\mathrm{TH}$-positive cells. Compared with the control group, TH-positive cells were significantly increased in NSCs cultured with PTE (Figure 2(c)).

\subsection{Effect of PTE on Global $5 \mathrm{hmC}$ Levels and TET1 Expression} during NSC Differentiation. To determine the effect of PTE on DNA demethylation during NSC differentiation, we examined the expression of global $5 \mathrm{hmC}$ levels by immunofluorescence (Figure 3(a)). Our results showed that, during NSC differentiation, global $5 \mathrm{hmClevels}$ were significantly increased in the PTE group compared with the control group. Simultaneously, we performed a dot blot analysis to detect $5 \mathrm{hmC}$ levels (Figure 3(b)). The expression of $5 \mathrm{hmC}$ levels was significantly increased in the PTE group compared with the control group.

Next, we examined the expression of TET1 in total protein and nuclear protein extracts by western blot analysis (Figure 3(c)). As expected, TET1 expression in the total protein extract was significantly upregulated in the PTE group compared with the control group (Figure 3(d)). Further, TET1 expression in the nuclear extract was also higher in the PTE group compared with the control group (Figure 3(e)). Additionally, increased Tet1 mRNA levels were detected in the PTE group (Figure 3(f)). Altogether, these results show that PTE can upregulate the expression of TET1 and 5hmC levels, which may regulate NSC differentiation.
3.4. Both PTE and PT Enhancing the FoxA2 Expression. To investigate which transcription factor is involved in the NSC differentiation induced by PTE, we focused on FoxA2, a critical transcription factor for dopaminergic neurons. Therefore, we examined the effect of PTE on FoxA2 expression during NSC differentiation, ex vivo. The results showed that mRNA levels of Foxa2 in the PTE group were significantly increased as compared with the control group (Figure 4(a)). Further, the expression of FoxA2 in total protein and nuclear protein was detected by western blot analysis (Figure 4(b)). Results showed that the expression of FoxA2 was significantly increased in the PTE group in total protein and nuclear protein extracts (Figures 4(c) and 4(d)). Moreover, we examined the expression of FoxA2 in brain tissue sections and observed a significant increase in FoxA2 expression in the high-dose drug and low-dose drug groups compared with the PD model group (Figures 4(e) and 4(f)).

3.5. Effect of PTE on the Interaction between TET1 and FoxA2. To investigate the mechanism of PTE on TET1 and FoxA2 during NSC differentiation, we performed immunofluorescence colocalization of TET1 and FoxA2. The results showed that expressions of TET1 and FoxA2 were significantly increased in the nucleus in the PTE group compared with the control group (Figure 5(a)). Subsequently, we performed coimmunoprecipitation (Co-IP) to further examine the relationship between FoxA2 and TET1 protein (Figure 5(b)). Interestingly, we detected an interaction between TET1 protein and FoxA2 during NSC differentiation into dopaminergic neurons. Specifically, an increase in the combination rate of TET1 and FoxA2 was found in the PTE group compared with the control group (Figure 5(c)).

3.6. Both TET1 and FoxA2 Are Indispensable for NSC Differentiation into Dopaminergic Neurons. Based on our results, we hypothesized that TET1 and FoxA2 are required for the differentiation of NSCs into dopaminergic neurons. Next, we used small interfering RNA (siRNA) to silence Tet 1 or Foxa 2 during the first few days of NSC differentiation. The silencing efficiency was evaluated (Figures 6(a) and 6(b)). Based on the silencing efficiency, we selected siTet1_001 and siFoxa2_002 for further experiments. Our subsequent results showed that Foxa2 mRNA levels did not change when Tet1 was silenced, yet the expression of the $T h$ gene was 

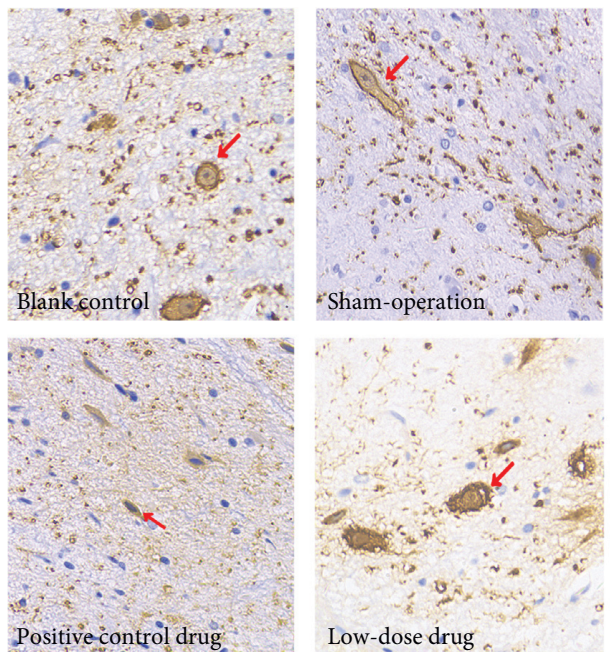

(a)
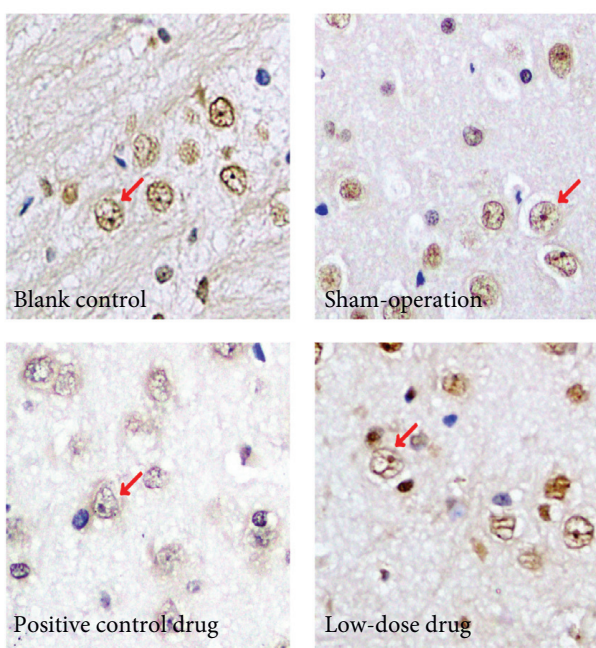

(c)
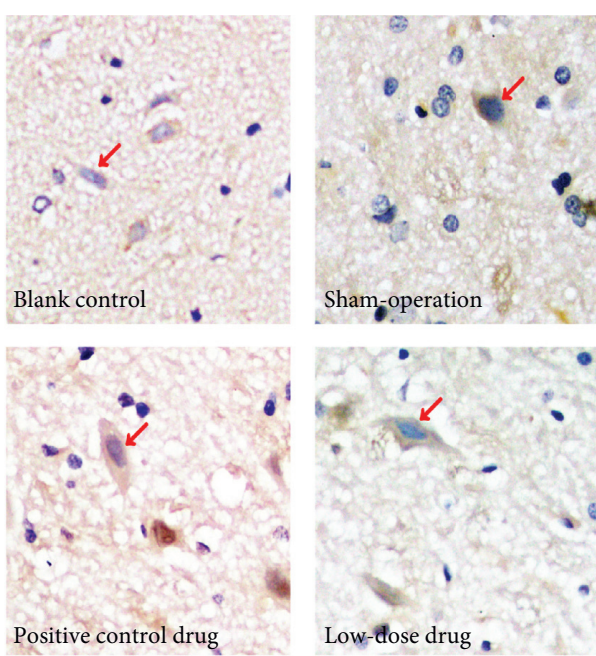

(e)

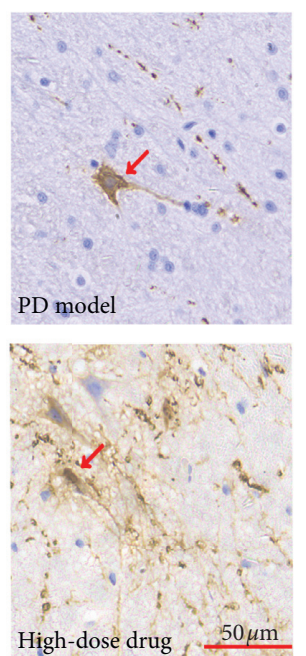

High-dose drug, $50 \mu \mathrm{m}$
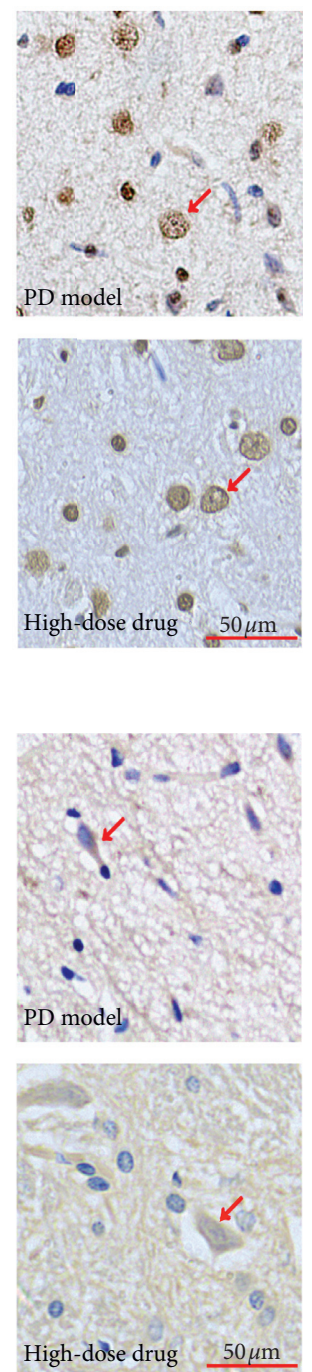

High-dose drug $\quad 50 \mu \mathrm{m}$

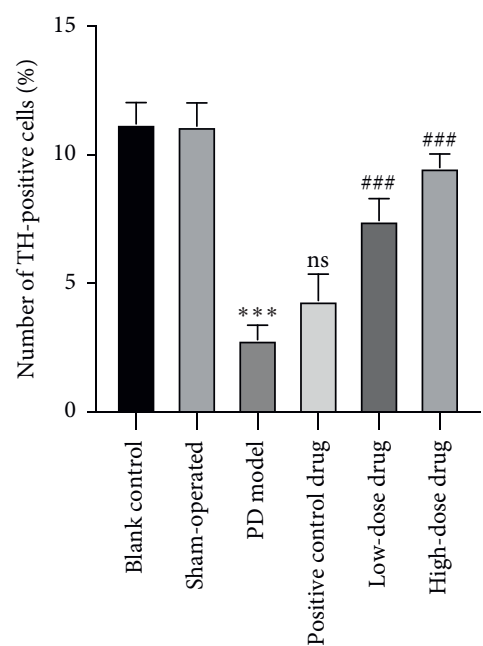

(b)

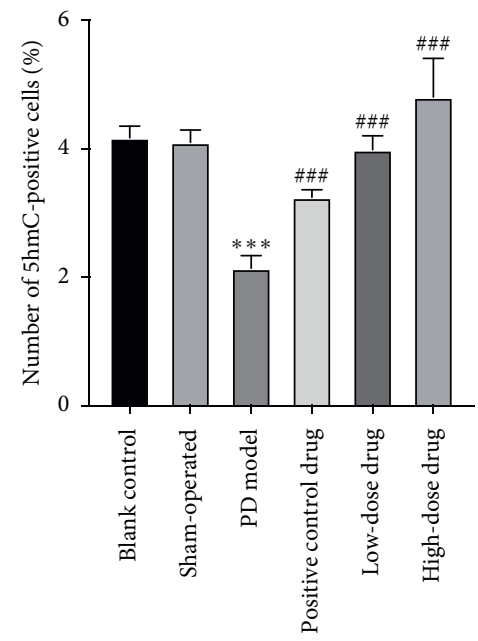

(d)

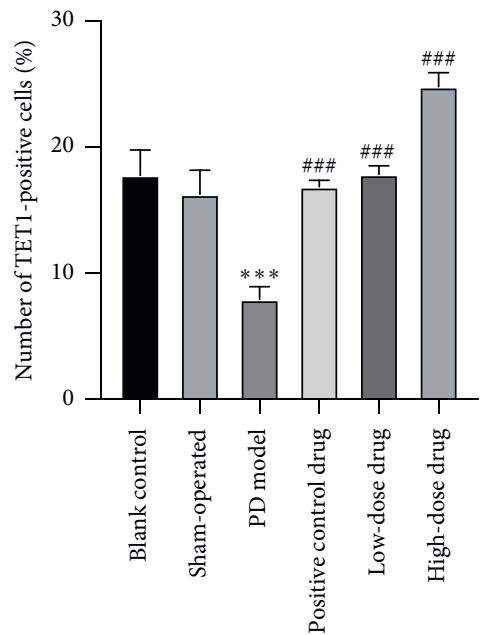

(f)

FIGURe 1: Effects of PT on substantia nigra (SN) of midbrain for PD rats (400x). (a) Staining of TH in rat brain (SN) tissue sections by IHC analysis (magnification: 400x). (b) Statistical analysis of the positive rate of TH-positive cells by using ImageJ software. (c) Staining of 5hmC in rat brain (SN) tissue sections by IHC analysis (magnification: 400x). (d) Statistical analysis of the positive rate of 5hmC-positive cells by using ImageJ software. (e) Staining of TET1 in rat brain (SN) tissue sections by IHC analysis (magnification: 400x). (f) Statistical analysis of the positive rate of TET1-positive cells by using ImageJ software. The positive cells were stained brown, marked by red arrow $(\uparrow)$. ${ }^{* * *} P<0.01$ compared with blank control; ${ }^{\# \# \#} P<0.01$ compared with PD model. ns: not significant; $P: P$ values. The description of the statistical analyses is provided in Supplementary Table S1. 


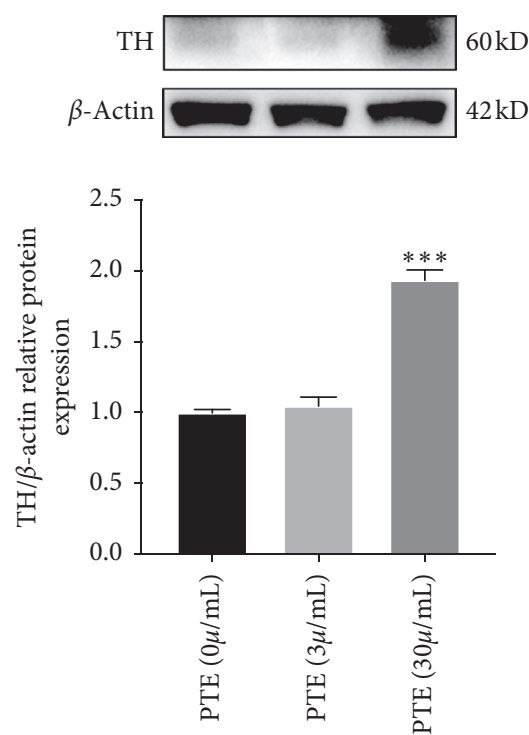

(a)
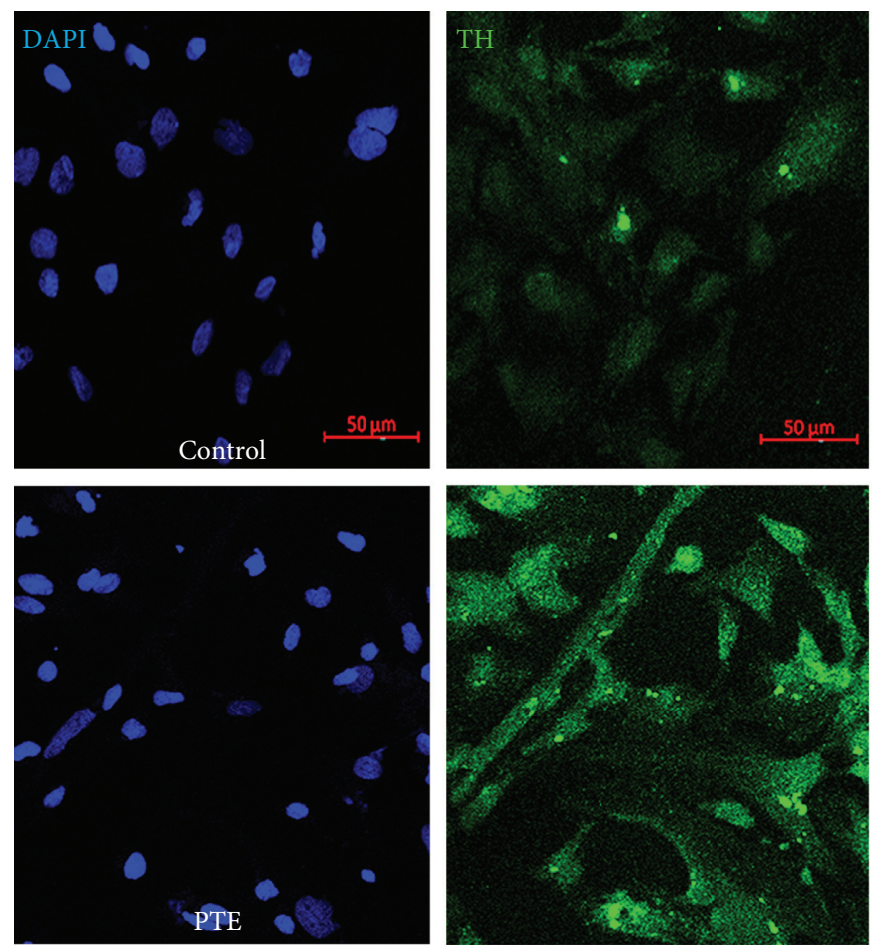

(c)

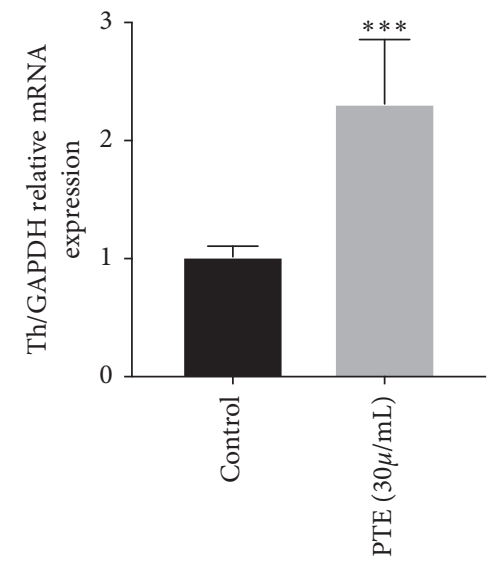

(b)
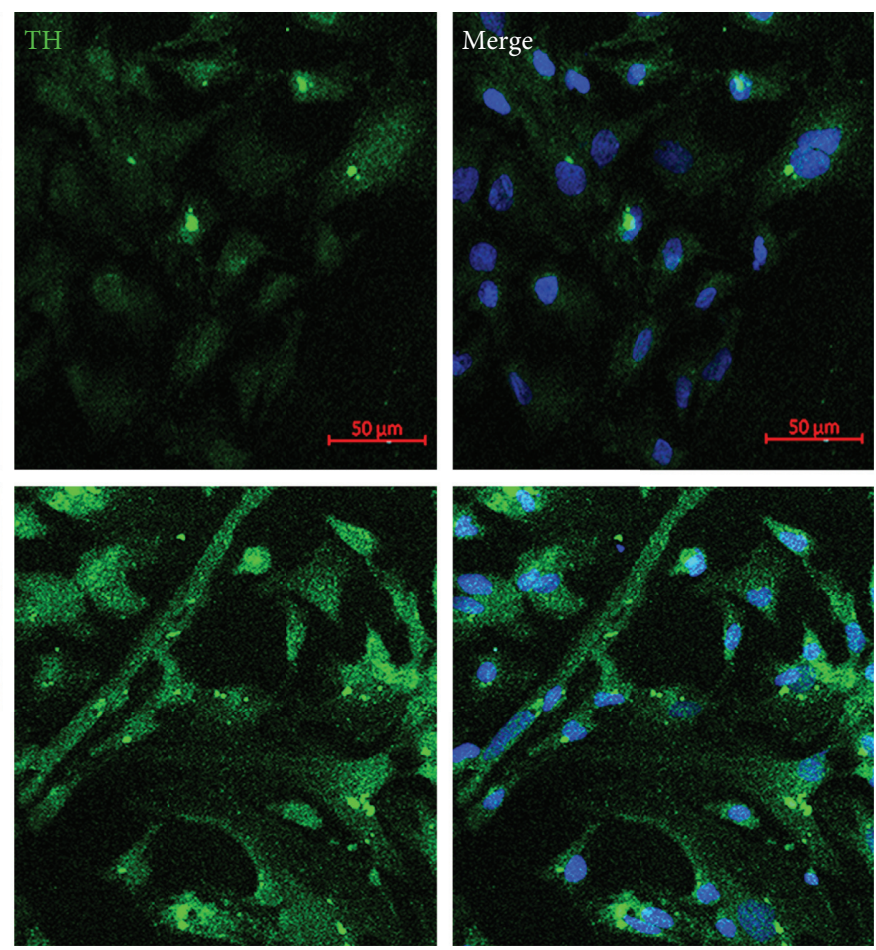

Figure 2: Effects of PTE on TH expression. (a) Western blot analysis for dopaminergic marker tyrosine hydroxylase (TH). ${ }^{* * *} P<0.01$ compared with PTE $(0 \mu \mathrm{g} / \mathrm{ml})$. (b) Expression of mRNA level of Th by quantitative real-time PCR (qRT-PCR) analysis, PTE (30 $\mu \mathrm{g} / \mathrm{ml})$. (c) Immunofluorescence staining of TH expression. Staining is shown for TH (green), with DAPI for nuclear staining (scale bar: $50 \mu \mathrm{m}$ ). NSCs were differentiated into dopaminergic neurons cultures with PTE $(30 \mu \mathrm{g} / \mathrm{ml})$ for 5 days. ${ }^{* * *} P<0.01$ compared with control. $P: P$ values. The description of the statistical analyses is provided in Supplementary Tables S2 and S3.

significantly downregulated (Figure 6(c)). Additionally, we found that following the silencing of Tet1 in the PTE group, expression of Th was not significantly different compared with the siTet1 group (Figure 6(d)). Similarly, after silencing of Foxa2, there was no change in Tet1 expression, while Th expression was also significantly decreased (Figure 6(e)). These results show no significant differences in Th expression in the PTE group compared with the siFoxa2 group, after Foxa2 had silenced (Figure 6(f)).

\section{Discussion}

Currently, treatments for PD are still limited. Clinical treatment of PD is mainly based on drug therapy. Levodopa 

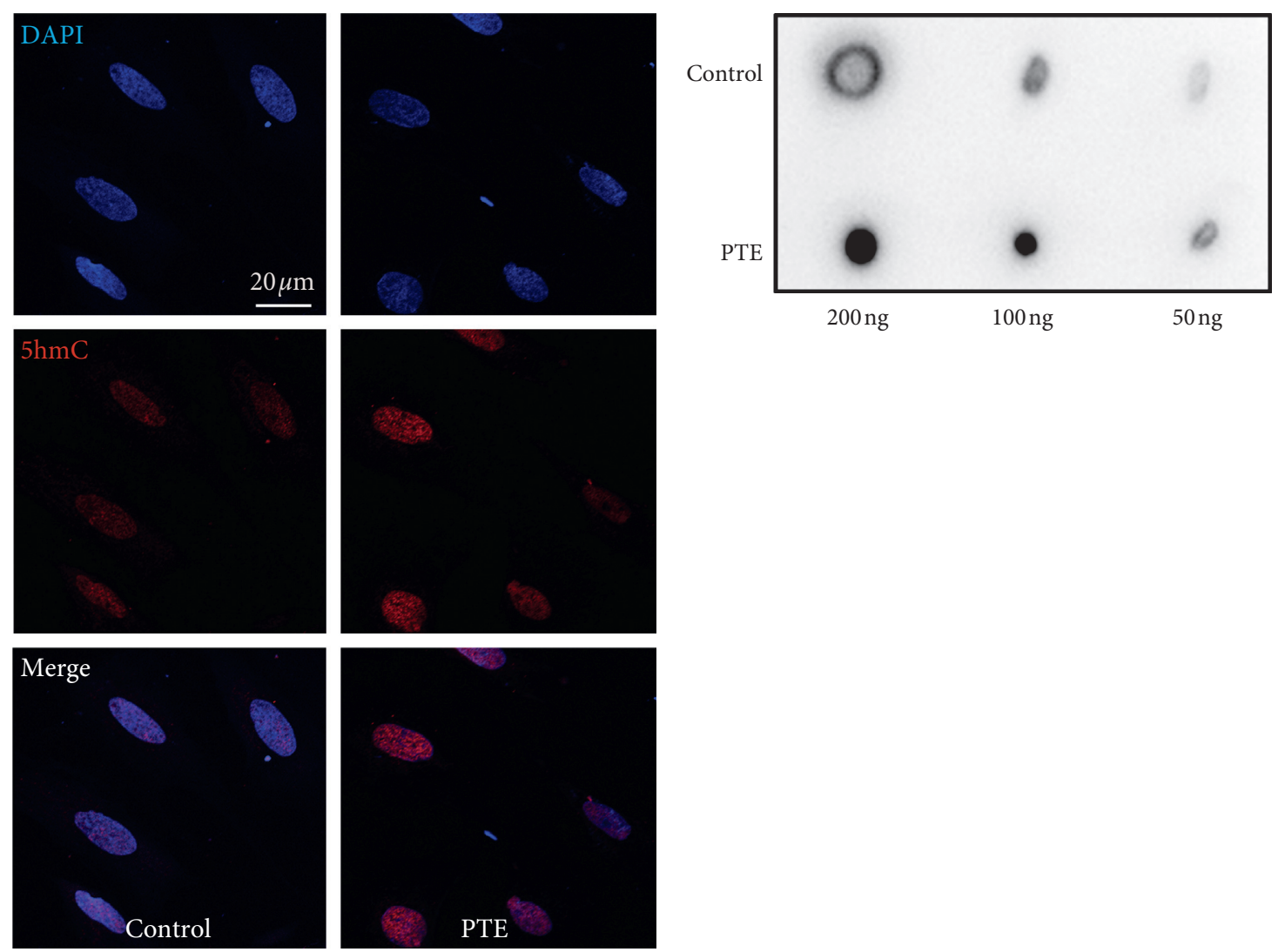

(a)

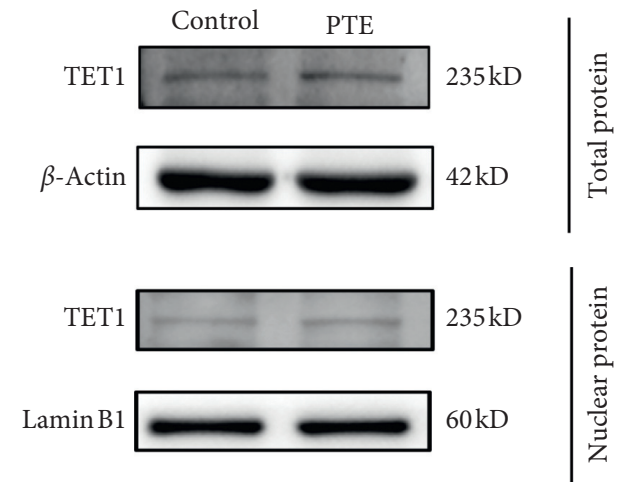

(b)

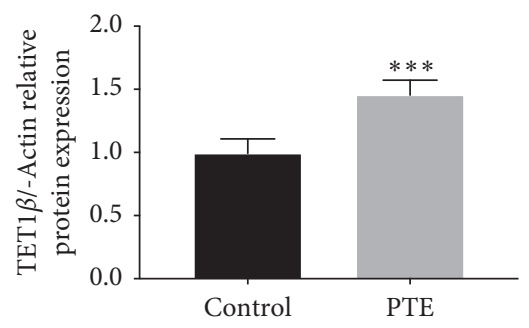

(d)

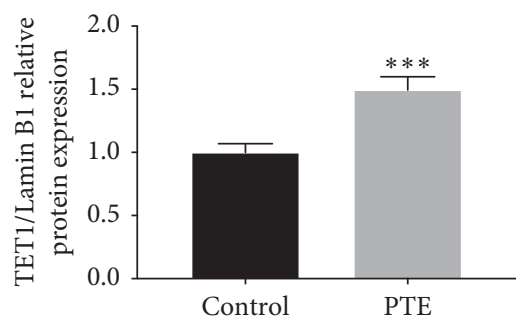

(e)

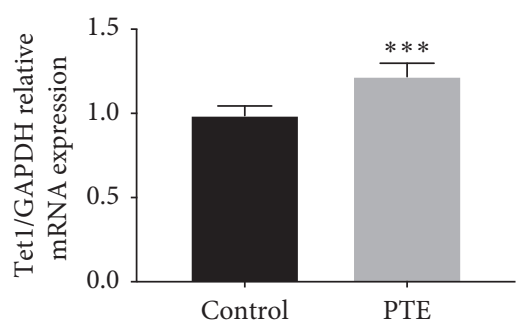

(f)

FIGURE 3: Effect of PTE on global 5hmC levels and TET1 expression. (a) Immunofluorescence staining of 5hmC expression. Staining is shown for $5 \mathrm{hmC}$ (red), with DAPI for nuclear staining (scale bar: $20 \mu \mathrm{m}$ ). (b) Dot blot analysis of $5 \mathrm{hmC} \mathrm{level.} \mathrm{(c)} \mathrm{Expression} \mathrm{of} \mathrm{TET1} \mathrm{in} \mathrm{total}$ protein and nuclear protein by western blot analysis. (d) Statistical analysis of gray value of TET1 expression in total protein. (e) Statistical analysis of gray value of TET1 expression in nuclear protein. (f) Expression of mRNA levels of Tet1 by qRT-PCR. ${ }^{* * *} P<0.01$ compared with control. $P$ : $P$ values. The description of the statistical analyses is provided in Supplementary Table S4. 


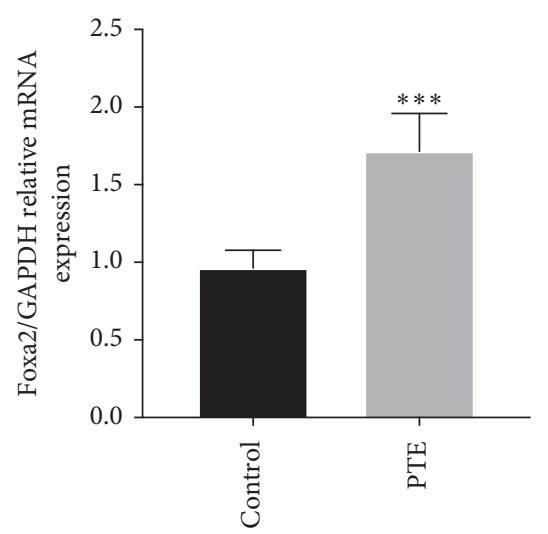

(a)

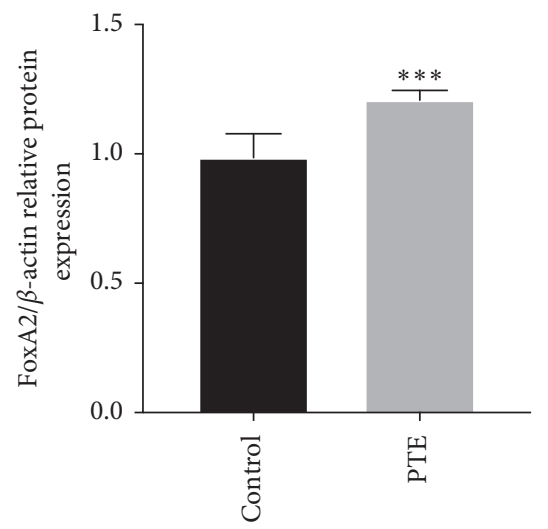

(c)
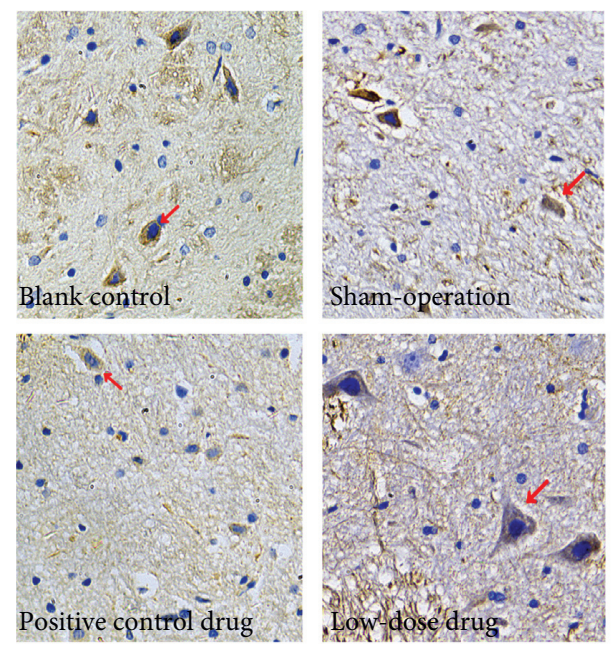

(e)
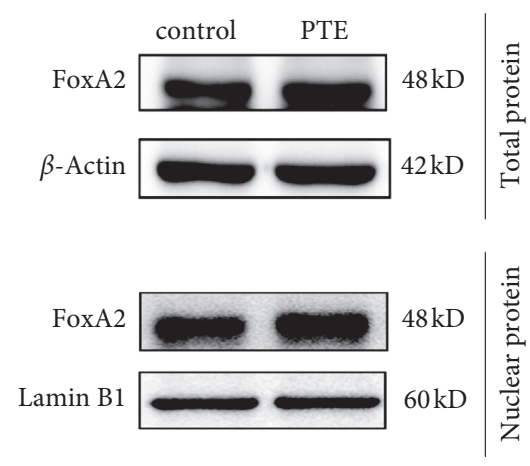

(b)

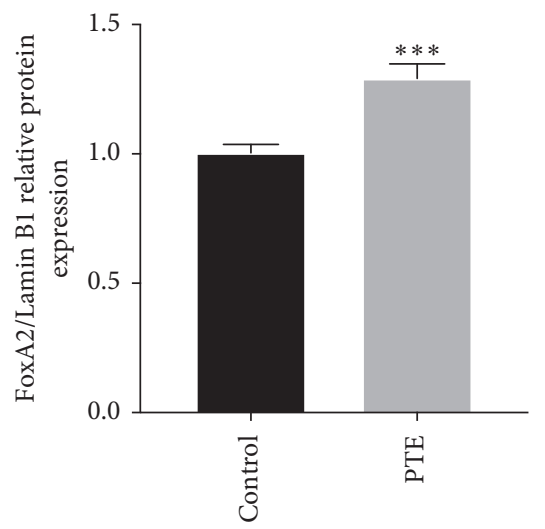

(d)

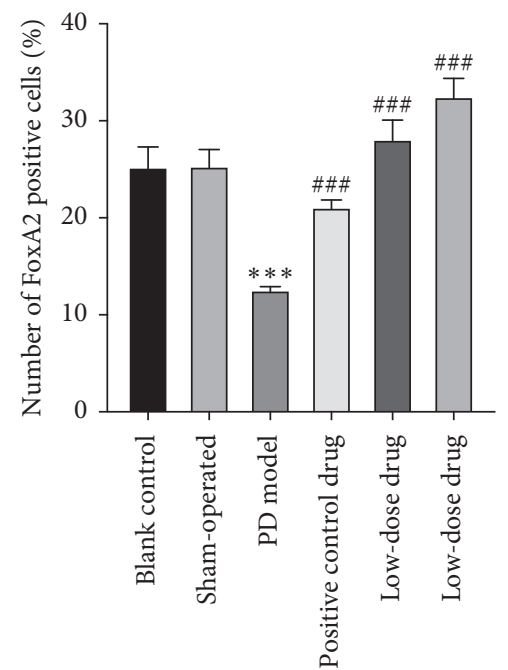

(f)

FIgURE 4: Effect of PTE and PT on FoxA2 expression. (a) Expression of mRNA levels of Foxa2 by qRT-PCR. (b) Expression of FoxA2 in total protein and nuclear protein by western blot analysis. (c) Statistical analysis of gray value of FoxA2 expression in total protein. (d) Statistical analysis of gray value of FoxA2 expression in nuclear protein. ${ }^{* * *} P<0.01$ compared with control. (e) Staining of FoxA2 in rat brain (SN) tissue sections by IHC analysis (magnification: 400x). (f) Statistical analysis of the positive rate of FoxA2-positive cells by using ImageJ software. The positive cells were stained brown, marked by red arrow $(\uparrow) .{ }^{* * *} P<0.01$ compared with blank control, \#\#\# $P<0.01$ compared with PD model. $P$ : $P$ values. The description of the statistical analyses is provided in Supplementary Tables S1 and S5.

is considered to be an effective drug for treating PD but can only alleviate the symptoms of patients with $\mathrm{PD}$ without achieving a cure. Finding a more effective therapy is an urgent task. Based on the current situation, we are concerned about stem cell therapy, which is considered to be a promising treatment. Moreover, if transplanted stem cells are used to treat PD, complications may arise such as low cell survival rate, poor long-term efficacy, and inability to 

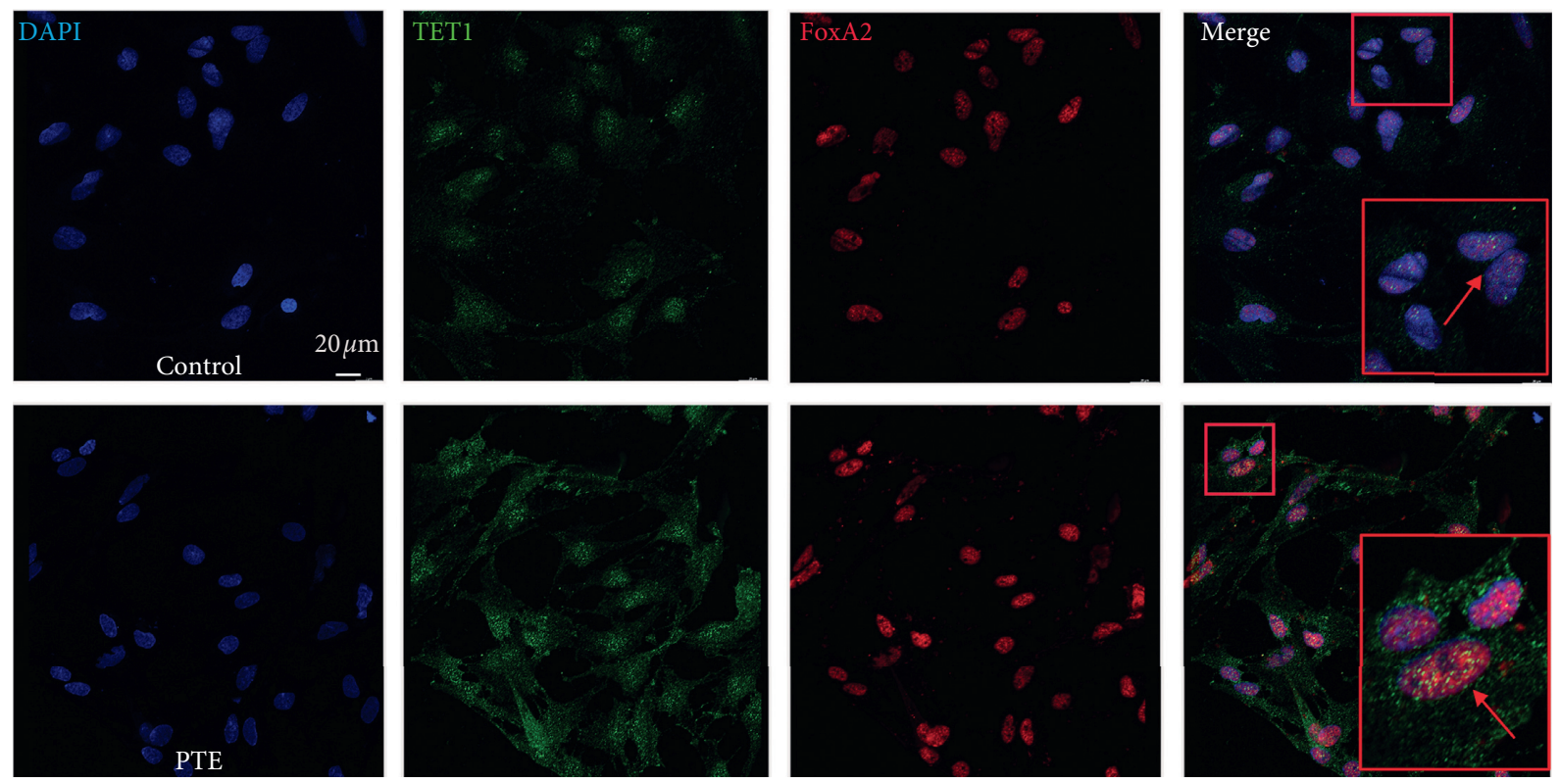

(a)

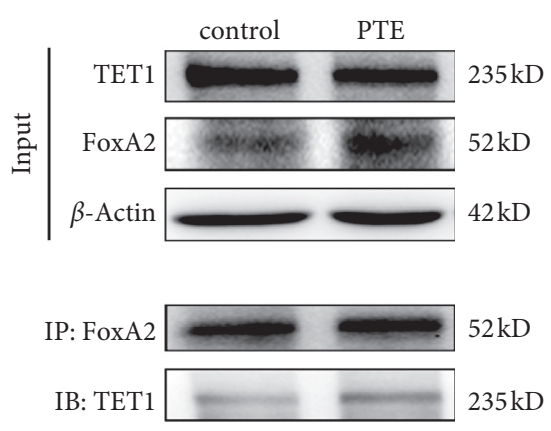

(b)

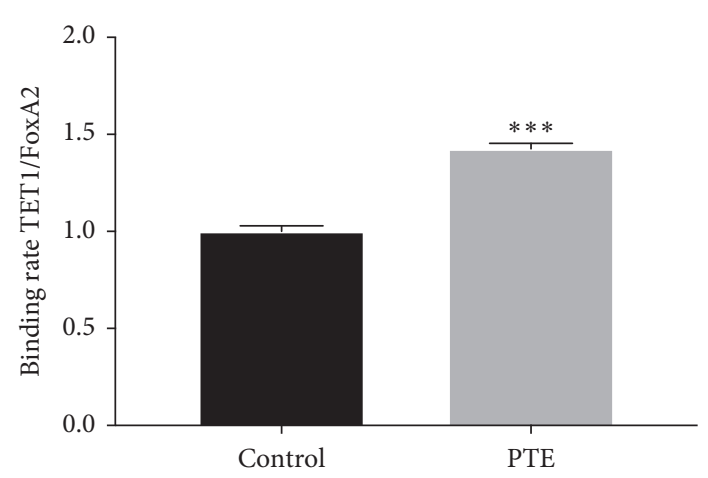

(c)

FIGURE 5: Effect of PTE on the interaction between TET1 and FoxA2. (a) Immunofluorescence colocation staining of TET1 and FoxA2 expression. Staining is shown for TET1 (green) and FoxA2 (red), with DAPI for nuclear staining (scale bar: $20 \mu \mathrm{m}$ ). (b) Coimmunoprecipitation (Co-IP) of TET1 and FoxA2 was assessed by western blot analysis. (c) Statistical analysis of gray value of binding rate between TET1 and FoxA2. ${ }^{* *} P<0.01$ compared with control. $P: P$ values. The description of the statistical analyses is provided in Supplementary Table S6.

differentiate directionally. Compared with these caveats, because of the multitarget effects of TCM, this approach has advantages of less toxicity and side effects. Consequently, if PTE can effectively promote NSCs to directionally differentiate into dopaminergic neurons, it will provide more effective and low-cost therapy for PD.

The 6-OHDA-induced rat model of PD causes a significant decrease in the expression of $\mathrm{TH}$, which we used here as a marker to investigate the role of PT. In our study, different outcomes were shown with levodopa or PT treatment. Since levodopa is a raw material that can be used for synthesizing dopamine, its therapeutic mechanism is well known [27]. However, the mechanism of PT is not well known. Nevertheless, because dopamine function could be restored, it is likely that $\mathrm{TH}$ expression is upregulated. Hence, we suspect that PT may have an active effect on promoting NSC differentiation into dopaminergic neurons.
After PT treatment, the TET1 enzyme and $5 \mathrm{hmC}$ results showed an effect on promoting NSC differentiation into dopaminergic neurons, possibly by mediating DNA demethylation. A previous study showed an increase in the percentage of NSC differentiation into dopaminergic neurons, which was closely associated with an increase in $5 \mathrm{hmC}$ levels mediated by TET1 [28]. This suggests that TET1 and TET-mediated $5 \mathrm{hmC}$ modification are important for the differentiation of NSCs.

To determine the mechanism by which PT can enhance NSCs to differentiate into dopaminergic neurons, we observed the effect of PTE on the differentiation of NSCs ex vivo. Our previous work found that PTE, which is the main active ingredient of PT, could increase the number of $\mathrm{TH}$ positive cells differentiated from NSCs [29]. Moreover, our ex vivo experiments show that PTE can effectively enhance NSC differentiation into dopaminergic neurons and 


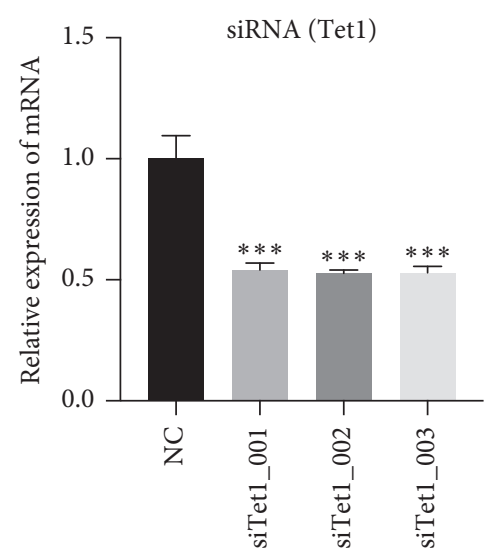

(a)

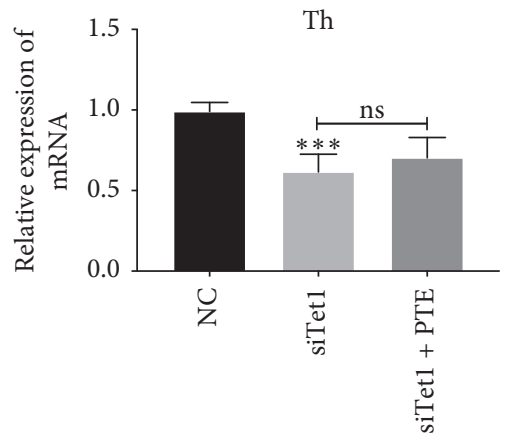

(d)

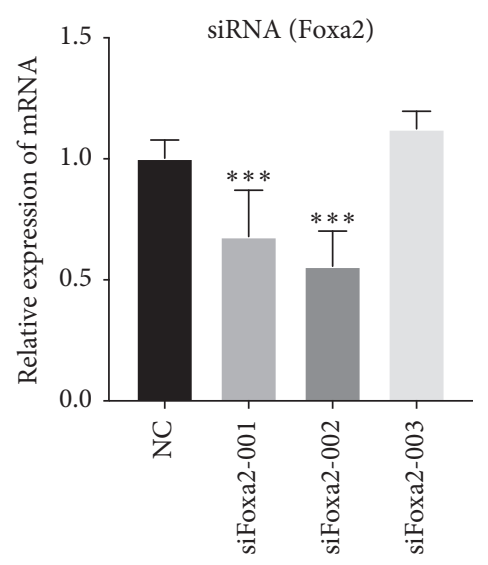

(b)

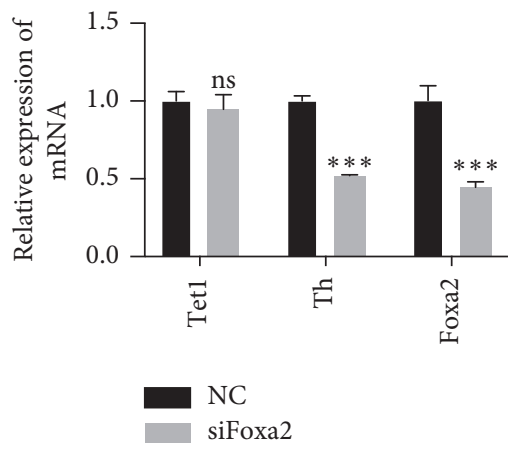

(e)

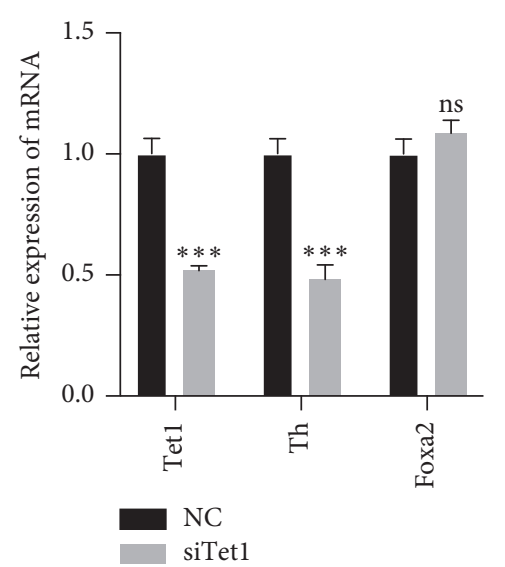

(c)

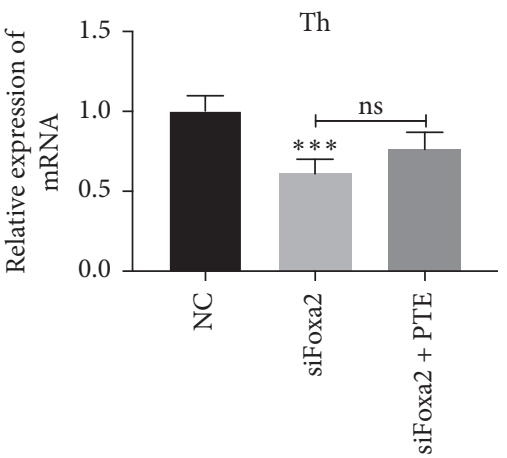

(f)

FIgURE 6: siRNA silencing of Tet1 and Foxa2 gene. (a) siRNA silencing of Tet1 gene. The expression of Tet1 was assessed by qRT-PCR, and the efficiency of silencing was evaluated. (b) siRNA silencing of Foxa2 gene. The expression of Foxa2 was assessed by qRT-PCR. (c) Silencing of Tet1 was done with interference fragment siTet1_001, and the expression of mRNA level of Tet1, Th, and Foxa2 was assessed. (d) Expression of mRNA level of Th by qRT-PCR analysis. siRNA silencing of Tet 1 was done in neural stem cells for $24 \mathrm{~h}$ and cells were cultured with PTE $(30 \mu \mathrm{g} / \mathrm{ml})$ for $24 \mathrm{~h}$. (e) Silencing of Foxa2 was done with interference fragment siFoxa2_002, and the expression of mRNA level of Tet1, Th, and Foxa2 was assessed. (f) Expression of mRNA level of Th by qRT-PCR analysis. At the differentiation stage, siRNA silencing of Foxa2 were done in neural stem cells for $24 \mathrm{~h}$ and cells were cultured with PTE $(30 \mu \mathrm{g} / \mathrm{ml})$ for $24 \mathrm{~h} .{ }^{* * *} P<0.01$ compared with control, NC: NControl, ns: not significant; $P$ : $P$ values. The description of the statistical analyses is provided in Supplementary Tables S7-S12.

significantly increase the number of dopaminergic neurons, consistent with in vivo experiments.

Our TET1 and 5hmC results suggest that PTE upregulates levels of DNA-5hmC modification, which may regulate NSC differentiation. Another study showed that TET protein interacts with transcriptional factors to regulate cell differentiation [30]. Consequently, we were interested in FoxA2, which is an essential transcriptional factor in the multiple phases of mDA neuronal development. FoxA2 regulates neurogenesis of $\mathrm{mDA}$ progenitors and early differentiation of $\mathrm{mDA}$ immature neurons, subsequently controlling $\mathrm{TH}$ expression in mature $\mathrm{mDA}$ neurons during late differentiation [31]. Further, the FoxA2 results suggest that in NSC differentiation into dopaminergic neurons during PTE treatment, FoxA2 may play a critical role. And, the result of in vivo experiment confirms that FoxA2 is increased after treatment with PT. Based on the results, it was suggesting that increasing FoxA2 and TET1 may promote NSCs to differentiate into dopaminergic neurons. Hence, these effects of PTE are likely to be achieved by upregulating FoxA2 and TET1.
However, downregulating FoxA2 may lead to loss of the differentiation potential of ventral mesencephalonderived neural precursor cells to differentiate into dopaminergic neurons, while activation of endogenous FoxA2 gene by epigenetic regulation may promote dopaminergic neuron production [32]. Furthermore, the positions of FoxA2 binding sites have been identified on the TH promoter, with FoxA2 able to directly activate $\mathrm{TH}$ expression in $\mathrm{mDA}$ progenitors and mature neurons [33].

In the present study, we show an interplay between TET1 and FoxA2 during NSC differentiation into dopaminergic neurons. PTE enhanced the combination of TET1 and FoxA2, which activated TH expression and promoted NSC differentiation into dopaminergic neurons. Hence, it could be hypothesized that after binding to the $\mathrm{TH}$ promoter, FoxA2 guides TET1 to catalyze $5 \mathrm{mC}$ to $5 \mathrm{hmC}$, enhancing the transcription of $\mathrm{TH}$ and thereby regulating NSC differentiation into dopaminergic neurons.

Finally, we predict that the efficacy of PT for the treatment of $\mathrm{PD}$ in vivo may be achieved by promoting 
endogenous NSCs to directionally differentiate into dopaminergic neurons through regulation of TET1 and FoxA2.

\section{Conclusions}

The present study further clarifies the underlying mechanism by which PTE promotes NSC differentiation. Taken together, PTE has active effects in promoting NSCs to directly differentiate into dopaminergic neurons. Further, PTE increases the efficiency of directional differentiation of NSCs into dopaminergic neurons by increasing the binding rate of TET1 and FoxA2.

\section{Abbreviations}

PT: $\quad$ Plastrum Testudinis

PTE: $\quad$ Ethyl acetate extracts from Plastrum Testudinis

NSCs: Neural stem cells

6-OHDA: 6-Hydroxydopamine

TH: Tyrosine hydroxylase

TET1: Ten-eleven translocation 1

FoxA2: $\quad$ Forkhead box A2

5hmC: 5-Hydroxymethylcytosine

5mC: 5-Methylcytosine

TCM: Traditional Chinese medicine

SN: $\quad$ Substantia nigra

mDA: $\quad$ Midbrain dopamine.

\section{Data Availability}

All data generated or analyzed during this study are included in this published article. The data used to support the findings of our study are available from the corresponding author upon request.

\section{Conflicts of Interest}

The authors declare that there are no conflicts of interest regarding the publication of this paper.

\section{Authors' Contributions}

Jun Zhong was responsible for conceptualization, investigation, validation, writing of the original draft; Sen Ye conceptualized the study and reviewed and edited the manuscript; Xiaoli Zhou investigated the study; Jiapei Huang validated the data; Xican Li and Saixia Zhang contributed resources; Jianhong Zhou investigated the study; Dongfeng Chen supervised the study; Caixia Li conceptualized the study, reviewed and edited the manuscript, and involved in project administration. All authors read and approved the final manuscript.

\section{Acknowledgments}

The authors thank Rachel James, Ph.D., from Liwen Bianji, Edanz Group China (http://www.liwenbianji.cn/ac), for editing the English text of a draft of this manuscript. This work was supported by grants from the National Natural Science Foundations of China (no. 81303116), the
Guangdong Provincial Science \& Technology Project (no. 2016A020226017), and Science Program for Overseas Scholar of Guangzhou University of Chinese Medicine (no. XH20170103).

\section{Supplementary Materials}

In this supplementary material, the statistical analyses in the description of the results have provided the means, standard deviations, and $P$ values for each figure caption. (Supplementary Materials)

\section{References}

[1] S. L. Kowal, T. M. Dall, R. Chakrabarti, M. V. Storm, and A. Jain, "The current and projected economic burden of parkinson's disease in the United States," Movement Disorders, vol. 28, no. 3, pp. 311-318, 2013.

[2] F. Sorbo and A. Albanese, "Levodopa-induced dyskinesias and their management," Journal of Neurology, vol. 255, no. S4, pp. 32-41, 2008.

[3] Y. Qian, X. X. Chen, W. Wang et al., "Transplantation of Nurr1-overexpressing neural stem cells and microglia for treating parkinsonian rats," CNS Neuroscience \& Therapeutics, vol. 26, no. 1, pp. 55-65, 2019.

[4] R. Wijeyekoon and R. A. Barker, "Cell replacement therapy for parkinson's disease," Biochimica et Biophysica Acta (BBA)-Molecular Basis of Disease, vol. 1792, no. 7, pp. 688-702, 2009.

[5] F. H. Gage, "Mammalian neural stem cells," Science, vol. 287, no. 5457, pp. 1433-1438, 2000.

[6] S. Suksuphew and P. Noisa, "Neural stem cells could serve as a therapeutic material for age-related neurodegenerative diseases," World Journal of Stem Cells, vol. 7, no. 2, pp. 502-511, 2015.

[7] H. Tao, P. Xie, Y. Cao et al., "The dynamic DNA demethylation during postnatal neuronal development and neural stem cell differentiation," Stem Cells International, vol. 2018, Article ID 2186301, 10 pages, 2018.

[8] H. Zhou, B. Wang, H. Sun, X. Xu, and Y. Wang, "Epigenetic regulations in neural stem cells and neurological diseases," Stem Cells International, vol. 2018, Article ID 6087143, 10 pages, 2018.

[9] K. P. Koh, A. Yabuuchi, S. Rao et al., "Tet1 and Tet2 regulate 5hydroxymethylcytosine production and cell lineage specification in mouse embryonic stem cells," Cell Stem Cell, vol. 8, no. 2, pp. 200-213, 2011.

[10] X. Wu and Y. Zhang, "TET-mediated active DNA demethylation: mechanism, function and beyond," Nature Reviews Genetics, vol. 18, no. 9, pp. 517-534, 2017.

[11] Y. Cheng, A. Bernstein, D. Chen, and P. Jin, "5-hydroxymethylcytosine: a new player in brain disorders?” Experimental Neurology, vol. 268, pp. 3-9, 2015.

[12] J.-P. Etchegaray, L. Chavez, Y. Huang et al., "The histone deacetylase SIRT6 controls embryonic stem cell fate via TETmediated production of 5-hydroxymethylcytosine," Nature Cell Biology, vol. 17, no. 5, pp. 545-557, 2015.

[13] M. A. Hahn, R. Qiu, X. Wu et al., "Dynamics of 5-hydroxymethylcytosine and chromatin marks in Mammalian neurogenesis," Cell Reports, vol. 3, no. 2, pp. 291-300, 2013.

[14] H. Jiang, Z. Xu, P. Zhong et al., "Cell cycle and p53 gate the direct conversion of human fibroblasts to dopaminergic 
neurons," Nature Communications, vol. 6, no. 1, p. 10100, 2015.

[15] P.-B. Ancey, S. Ecsedi, M.-P. Lambert et al., "TET-catalyzed 5hydroxymethylation precedes HNF4A promoter choice during differentiation of bipotent liver progenitors," Stem Cell Reports, vol. 9, no. 1, pp. 264-278, 2017.

[16] S.-L. Ang, "Foxa1 and Foxa2 transcription factors regulate differentiation of midbrain dopaminergic neurons," Advances in Experimental Medicine and Biology, vol. 651, pp. 58-65, 2009.

[17] S. R. W. Stott, E. Metzakopian, W. Lin, K. H. Kaestner, R. Hen, and S.-L. Ang, "Foxa1 and foxa2 are required for the maintenance of dopaminergic properties in ventral midbrain neurons at late embryonic stages," Journal of Neuroscience, vol. 33, no. 18, pp. 8022-8034, 2013.

[18] S. C. Daubner, T. Le, and S. Wang, "Tyrosine hydroxylase and regulation of dopamine synthesis," Archives of Biochemistry and Biophysics, vol. 508, no. 1, pp. 1-12, 2011.

[19] W. Qin, S. Chen, S. Yang, Q. Xu, C. Xu, and J. Cai, "The effect of traditional Chinese medicine on neural stem cell proliferation and differentiation," Aging and Disease, vol. 8, no. 6, pp. 792-811, 2017.

[20] T. Shu, M. Pang, L. Rong et al., "Effects of Salvia miltiorrhiza on neural differentiation of induced pluripotent stem cells," Journal of Ethnopharmacology, vol. 153, no. 1, pp. 233-241, 2014.

[21] M. Torres-Pérez, R. I. Tellez-Ballesteros, L. Ortiz-López et al., "Resveratrol enhances neuroplastic changes, including hippocampal neurogenesis, and memory in balb/C mice at six months of age," PLoS One, vol. 10, no. 12, Article ID e0145687, 2015.

[22] Q. Shang, X. Yu, H. Ren et al., "Effect of plastrum testudinis extracts on the proliferation and osteogenic differentiation of rBMSCs by regulating p38 MAPK-related genes," EvidenceBased Complementary and Alternative Medicine, vol. 2019, Article ID 6815620, 10 pages, 2019.

[23] G. Mercanti, G. Bazzu, and P. Giusti, "A 6-hydroxydopamine in vivo model of parkinson's disease," Neurotrophic Factors, vol. 846, pp. 355-364, 2012.

[24] D. F. Chen, H. P. Zeng, S. H. Du et al., "Extracts from plastrum testudinis promote proliferation of rat bone-marrow-derived mesenchymal stem cells," Cell Proliferation, vol. 40, no. 2, pp. 196-212, 2007.

[25] T.-T. Wang, W. Chen, H.-P. Zeng, and D.-F. Chen, "Chemical components in extracts from Plastrum testudinis with proliferation-promoting effects on rat mesenchymal stem cells," Chemical Biology \& Drug Design, vol. 79, no. 6, pp. 1049-1055, 2012.

[26] S. Ahmed, "The culture of neural stem cells," Journal of Cellular Biochemistry, vol. 106, no. 1, pp. 1-6, 2009.

[27] M. G. Murer, R. Raisman-Vozari, and O. Gershanik, "Levodopa in parkinson's disease: neurotoxicity issue laid to rest?” Drug Safety, vol. 21, no. 5, pp. 339-352, 1999.

[28] X.-B. He, M. Kim, S.-Y. Kim et al., "Vitamin C facilitates dopamine neuron differentiation in fetal midbrain through TET1- and JMJD3-dependent epigenetic control manner," Stem Cells, vol. 33, no. 4, pp. 1320-1332, 2015.

[29] L. Meng, "Regulation of stem cell differentiate into dopaminergic nerve by extracts from plastrum testudinis promote," M.Sc. thesis, Guangzhou University of Chinese Medicine, Guangzhou, China, 2008.

[30] X. Li, B. Yao, L. Chen et al., "Ten-eleven translocation 2 interacts with forkhead box $\mathrm{O} 3$ and regulates adult neurogenesis," Nature Communications, vol. 8, no. 1, p. 15903, 2017.
[31] A. L. M. Ferri, W. Lin, Y. E. Mavromatakis et al., "Foxa1 and Foxa2 regulate multiple phases of midbrain dopaminergic neuron development in a dosage-dependent manner," Development, vol. 134, no. 15, pp. 2761-2769, 2007.

[32] S.-Y. Bang, S. H. Kwon, S.-H. Yi et al., "Epigenetic activation of the Foxa2 gene is required for maintaining the potential of neural precursor cells to differentiate into dopaminergic neurons after expansion," Stem Cells and Development, vol. 24, no. 4, pp. 520-533, 2015.

[33] W. Lin, E. Metzakopian, Y. E. Mavromatakis et al., "Foxa1 and Foxa2 function both upstream of and cooperatively with Lmxla and Lmxlb in a feedforward loop promoting mesodiencephalic dopaminergic neuron development," Developmental Biology, vol. 333, no. 2, pp. 386-396, 2009. 